M. Tsukamoto

Nagoya Math. J.

Vol. 194 (2009), 33-68

\title{
A PACKING PROBLEM FOR HOLOMORPHIC CURVES
}

\author{
MASAKI TSUKAMOTO
}

\begin{abstract}
We propose a new approach to the value distribution theory of entire holomorphic curves. We define packing density of Brody curves, and show that it has various non-trivial properties. The packing density of Brody curves can be considered as an infinite dimensional version of characteristic number, and it has an application to Gromov's mean dimension theory.
\end{abstract}

\section{$\S 1$. Main results}

\subsection{Introduction}

Since R. Nevanlinna discovered his celebrated theory on meromorphic functions ([14]), thousands of researchers have studied the value distribution theory of meromorphic functions and, more generally, entire holomorphic curves (and holomorphic mappings) in complex manifolds. This paper is a new approach to the value distribution theory. We define and study a packing problem for entire holomorphic curves.

Packing is usually a notion in discrete geometry. For example, the Kepler conjecture on the sphere packing in $\mathbb{R}^{3}$ is very famous. In this paper we define a packing density of an entire holomorphic curve and investigate its behavior. (To be precise, we consider only Brody curves.) In particular, we study entire holomorphic curves in the projective space and prove that their packing densities have a non-trivial upper bound. Hence their supremum becomes a non-trivial invariant of the projective space. This invariant can be considered as an infinite dimensional version of characteristic number, and it has an application to Gromov's mean dimension theory (see Subsection 1.5 and Tsukamoto [17]).

Received August 20, 2007.

Accepted August 5, 2008.

2000 Mathematics Subject Classification: 32H30.

This work was supported by Grant-in-Aid for JSPS Fellows (19.1530) from Japan Society for the Promotion of Science. 


\subsection{Packing density of Brody curves}

Let $X$ be a Hermitian manifold and $\omega$ be its Kähler form. Let $z=x+$ $y \sqrt{-1}$ be the natural coordinate in the complex plane $\mathbb{C}$. For a holomorphic map $f: \mathbb{C} \rightarrow X$, we define the pointwise norm $|d f|(z) \geq 0$ of the differential $d f$ by

$$
f^{*} \omega=|d f|^{2}(z) d x d y .
$$

This is the "dilatation" of the map $f$, i.e.,

$$
|d f|(z)=\sqrt{2}|d f(\partial / \partial z)|
$$

where $\partial / \partial z=\frac{1}{2}(\partial / \partial x-\sqrt{-1} \partial / \partial y)$ and the factor $\sqrt{2}$ comes from $|\partial / \partial z|=$ $1 / \sqrt{2}$ (the complex plane is equipped with the usual Euclidean metric). We call a holomorphic map $f: \mathbb{C} \rightarrow X$ a Brody curve if it satisfies $|d f| \leq 1$ (cf. Brody [2]). Let $\mathcal{M}(X, \omega)$ be the moduli space of Brody curves in $X$ :

$\mathcal{M}(X, \omega):=\{f: \mathbb{C} \rightarrow X \mid f$ is holomorphic and $|d f|(z) \leq 1$ for all $z \in \mathbb{C}\}$.

For a Brody curve $f \in \mathcal{M}(X, \omega)$, we define its packing density $\rho(f)$ by setting

$$
\rho(f):=\limsup _{R \rightarrow \infty} \frac{1}{\pi R^{2}} \int_{|z| \leq R} f^{*} \omega=\limsup _{R \rightarrow \infty} \frac{1}{\pi R^{2}} \int_{|z| \leq R}|d f|^{2} d x d y .
$$

This satisfies

$$
0 \leq \rho(f) \leq 1
$$

The integration of $f^{*} \omega$ is the energy functional. Hence if $\rho(f)$ is close to 1 , the energy of $f$ is densely packed in the complex plane. In other words, $\rho(f)$ evaluates the efficiency of the energy distribution of $f$. This is the reason why we call $\rho(f)$ "packing density". We define $\rho(X, \omega)$ by setting

$$
\rho(X, \omega):=\sup _{f \in \mathcal{M}(X, \omega)} \rho(f) .
$$

This satisfies

$$
0 \leq \rho(X, \omega) \leq 1
$$

Then we can define a packing problem. The packing problem for holomorphic curves is the problem of determining, or estimating, the value of $\rho(X, \omega)$. We will often abbreviate $\mathcal{M}(X, \omega)$ and $\rho(X, \omega)$ to $\mathcal{M}(X)$ and $\rho(X)$ when it causes no confusion. 
EXAMPLE 1.1. Let $\left(z_{1}, z_{2}, \ldots, z_{n}\right)$ be the natural coordinate system on $\mathbb{C}^{n}$. The Euclidean metric and its Kähler form on $\mathbb{C}^{n}$ are given by

$$
d s^{2}=\sum_{i=1}^{n} d z_{i} d \bar{z}_{i}, \quad \omega=\frac{\sqrt{-1}}{2} \sum_{i=1}^{n} d z_{i} \wedge d \bar{z}_{i} .
$$

Let $f: \mathbb{C} \rightarrow \mathbb{C}^{n}$ be the natural inclusion: $f(z):=(z, 0,0, \ldots, 0)$. It is obvious that $|d f| \equiv 1$. Hence $f \in \mathcal{M}\left(\mathbb{C}^{n}, \omega\right)$ and $\rho(f)=1$. Therefore

$$
\rho\left(\mathbb{C}^{n}, \omega\right)=1 .
$$

In the same way, if $X$ is a complex torus with the Euclidean metric induced by the universal covering, then we have

$$
\rho(X)=1 .
$$

Example 1.2. Let $\Delta=\{z \in \mathbb{C}|| z \mid<1\}$ be the unit disk and $g$ be an arbitrary Hermitian metric on $\Delta$. All holomorphic maps from $\mathbb{C}$ to $\Delta$ are constant maps by Liouville's theorem. Therefore we have

$$
\rho(\Delta, \omega)=0 .
$$

In the same manner, if $X$ is a compact Riemann surface of genus $\geq 2$, then we have

$$
\rho(X, \omega)=0 \text { for any Hermitian metric on } X .
$$

The above two examples are trivial extremal cases. Our main concern is the case of the complex projective space $\mathbb{C} P^{n}$ with the Fubini-Study metric $(n \geq 1)$. Let $\left[z_{0}: z_{1}: \cdots: z_{n}\right]$ be the homogeneous coordinate in $\mathbb{C} P^{n}$. We define the Fubini-Study metric form $\omega_{F S}$ by

$$
\omega_{F S}:=\frac{\sqrt{-1}}{2 \pi} \partial \bar{\partial} \log \left(1+\sum_{i=1}^{n}\left|z_{i}\right|^{2}\right) \quad \text { on }\left\{\left[1: z_{1}: \cdots: z_{n}\right]\right\} .
$$

$\omega_{F S}$ smoothly extends over $\mathbb{C} P^{n}$ and defines the Fubini-Study metric. This is normalized so that

$$
\int_{\mathbb{C} P^{1}} \omega_{F S}=1 \text { for } \mathbb{C} P^{1}:=\left\{\left[z_{0}: z_{1}: 0: \cdots: 0\right] \in \mathbb{C} P^{n}\right\} .
$$

The start point of the packing problem is the following: 
THEOREM 1.3.

$$
0<\rho\left(\mathbb{C} P^{n}, \omega_{F S}\right)<1,
$$

i.e., $\rho\left(\mathbb{C} P^{n}, \omega_{F S}\right)$ is not equal to 0 nor 1 .

This result means that the packing problem for holomorphic curves in $\mathbb{C} P^{n}$ is a non-trivial problem. (The essential point of this statement is that $\rho\left(\mathbb{C} P^{n}, \omega_{F S}\right)$ cannot be equal to 1.) We will usually abbreviate $\rho\left(\mathbb{C} P^{n}, \omega_{F S}\right)$ to $\rho\left(\mathbb{C} P^{n}\right)$.

Theorem 1.3 does not give an effective upper bound for $\rho\left(\mathbb{C} P^{n}\right)$. We investigate the explicit estimate for $\rho\left(\mathbb{C} P^{1}\right)$ in the next theorem.

THEOREM 1.4.

$$
\rho\left(\mathbb{C} P^{1}\right) \leq 1-10^{-100} .
$$

The above value, $1-10^{-100}$, itself has no importance. The important point is that it is an explicit number. $\left(\rho\left(\mathbb{C} P^{1}\right)\right.$ is a very transcendental object and it is far from obvious whether we can give an explicit estimate of it.) Actually the proof of this explicit estimate is the most difficult argument in this paper.

Next we study the behavior of $\rho\left(\mathbb{C} P^{n}\right)$ as $n$ goes to infinity. The natural inclusion $\mathbb{C} P^{n}=\left\{\left[z_{0}: z_{1}: \cdots: z_{n}: 0\right] \in \mathbb{C} P^{n+1}\right\} \hookrightarrow \mathbb{C} P^{n+1}$ is a holomorphic isometric imbedding. Hence we can consider

$$
\mathcal{M}\left(\mathbb{C} P^{1}\right) \subset \mathcal{M}\left(\mathbb{C} P^{2}\right) \subset \mathcal{M}\left(\mathbb{C} P^{3}\right) \subset \cdots \subset \mathcal{M}\left(\mathbb{C} P^{n}\right) \subset \mathcal{M}\left(\mathbb{C} P^{n+1}\right) \subset \cdots .
$$

It results that

$$
0<\rho\left(\mathbb{C} P^{1}\right) \leq \rho\left(\mathbb{C} P^{2}\right) \leq \rho\left(\mathbb{C} P^{3}\right) \leq \cdots \leq \rho\left(\mathbb{C} P^{n}\right) \leq \rho\left(\mathbb{C} P^{n+1}\right) \leq \cdots<1 .
$$

The following theorem determines the limit of this sequence.

THEOREM 1.5.

$$
\lim _{n \rightarrow \infty} \rho\left(\mathbb{C} P^{n}\right)=1 .
$$

\subsection{Gap theorem for holomorphic maps from elliptic curves}

As an application of packing problem, we can prove a "gap theorem" for elliptic functions and, more generally, holomorphic maps from elliptic curves to $\mathbb{C} P^{n}$.

Let $\mathbb{C} / \Lambda$ be a elliptic curve. Here $\Lambda$ is a lattice in $\mathbb{C}$. We give $\mathbb{C} / \Lambda$ the Euclidean metric induced by the universal covering $\mathbb{C}$. Let $f: \mathbb{C} / \Lambda \rightarrow \mathbb{C} P^{n}$ 
be a holomorphic map. (Here we don't consider any restriction on the norm $|d f|$.) We define the degree $\operatorname{deg}(f)$ by setting

$$
\operatorname{deg}(f):=\int_{\mathbb{C} / \Lambda} f^{*} \omega_{F S}=\int_{\mathbb{C} / \Lambda}|d f|^{2} d x d y .
$$

From the normalization of the Fubini-Study metric in $(2), \operatorname{deg}(f)$ is a nonnegative integer. From (3), we get an a priori estimate:

$$
\|d f\|_{\infty}^{2} \geq \frac{\operatorname{deg}(f)}{\operatorname{vol}(\mathbb{C} / \Lambda)} .
$$

Here $\|d f\|_{\infty}:=\sup _{z \in \mathbb{C} / \Lambda}|d f|(z)$ and $\operatorname{vol}(\mathbb{C} / \Lambda)$ denotes the volume of $\mathbb{C} / \Lambda$. The following result shows that this is not a best estimate.

TheOREM 1.6. For any holomorphic map $f: \mathbb{C} / \Lambda \rightarrow \mathbb{C} P^{n}$, we have

$$
\|d f\|_{\infty}^{2} \geq \frac{1}{\rho\left(\mathbb{C} P^{n}\right)} \frac{\operatorname{deg}(f)}{\operatorname{vol}(\mathbb{C} / \Lambda)} .
$$

From Theorem 1.3,

$$
\frac{1}{\rho\left(\mathbb{C} P^{n}\right)}>1
$$

Hence there exists a certain gap between the a priori estimate (4) and Theorem 1.6. The point is that $1 / \rho\left(\mathbb{C} P^{n}\right)$ is the universal constant which does not depend on any lattice $\Lambda$ nor any holomorphic map $f$.

\subsection{Packing problem for the complement of hyperplanes}

Next we study the packing problem for holomorphic curves in the complement of hyperplanes. Let $H_{0}, H_{1}, \ldots, H_{n}$ be the $n+1$ hyperplanes in $\mathbb{C} P^{n}$ defined by

$$
H_{i}: \quad \sum_{j=0}^{n} a_{i j} z_{j}=0 \quad(0 \leq i \leq n) .
$$

Here $\left[z_{0}: z_{1}: \cdots: z_{n}\right]$ is the homogeneous coordinate in $\mathbb{C} P^{n}$. Let $A:=$ $\left(a_{i j}\right)_{0 \leq i, j \leq n}$ be the coefficients matrix. $H_{0}, H_{1}, \ldots, H_{n}$ are said to be linearly independent if $A$ is a regular matrix.

TheOrem 1.7. Let $H_{0}, H_{1}, \ldots, H_{n}$ be $n+1$ linearly independent hyperplanes in $\mathbb{C} P^{n}$. Then

$$
\rho\left(\mathbb{C} P^{n} \backslash\left(H_{0} \cup H_{1} \cup \cdots \cup H_{n}\right), \omega_{F S}\right)=0 .
$$

Here we use the Fubini-Study metric as the metric on $\mathbb{C} P^{n} \backslash\left(H_{0} \cup \cdots \cup H_{n}\right)$. 
This theorem makes a sharp contrast with Theorem 1.3 and Theorem 1.5 .

Example 1.8. Consider the exponential function exp $: \mathbb{C} \rightarrow \mathbb{C} P^{1} \backslash$ $\{0, \infty\}$. This satisfies

$$
|d \exp |(z)=\frac{1}{\sqrt{\pi}} \frac{e^{x}}{1+e^{2 x}}<1, \quad(x=\operatorname{Re} z) .
$$

Hence it is an element of $\mathcal{M}\left(\mathbb{C} P^{1} \backslash\{0, \infty\}, \omega_{F S}\right)$. By a direct calculation (or using the above theorem), we can see

$$
\rho(\exp )=0
$$

PROBlem 1.9. If $n=1$, then we have a stronger result: It is known that all $f \in \mathcal{M}\left(\mathbb{C} P^{1} \backslash\{\infty\}, \omega_{F S}\right)$ has order $\leq 1$, where "order" means the order of the Nevanlinna characteristic function of $f$ (see Clunie-Hayman [4, Theorem 3], Minda [12, pp. 210-211] or Eremenko [6, Theorem 5.2]). Then

$$
\rho(f)=0 \text {. }
$$

Hence we have

$$
\rho\left(\mathbb{C} P^{1} \backslash\{\infty\}, \omega_{F S}\right)=0 .
$$

I don't know whether this result can be generalized to a higher dimensional case or not.

\subsection{Application to Gromov's mean dimension theory}

Packing density has a "moduli theoretic" meaning. (We don't prove the result of this subsection in this paper; For the proof and related arguments, see Tsukamoto [17].) Let $X \subset \mathbb{C} P^{n}$ be an algebraic set (not necessarily smooth), and set

$$
\mathcal{M}(X)=\mathcal{M}\left(X, \omega_{F S}\right):=\left\{f \in \mathcal{M}\left(\mathbb{C} P^{n}, \omega_{F S}\right) \mid f(\mathbb{C}) \subset X\right\} .
$$

We give the compact-open topology on $\mathcal{M}(X)$. Then $\mathcal{M}(X)$ becomes a compact (metrizable) space. We set

$$
\rho(X):=\sup _{f \in \mathcal{M}(X)} \rho(f) .
$$

From Theorem 1.3,

$$
\rho(X) \leq \rho\left(\mathbb{C} P^{n}\right)<1
$$


The Lie group $\mathbb{C}$ naturally acts on $\mathcal{M}(X)$ by

$$
\mathbb{C} \times \mathcal{M}(X) \longrightarrow \mathcal{M}(X), \quad(a, f(z)) \longmapsto f(z+a) .
$$

$\mathcal{M}(X)$ can be an infinite dimensional space, but we can consider its "mean dimension" $\operatorname{dim}(\mathcal{M}(X): \mathbb{C})$. Mean dimension is a "dimension of an infinite dimensional space" (see Gromov [7], Lindenstrauss-Weiss [11], Lindenstrauss [10] or Tsukamoto [17]). Packing density can be used to estimate the mean dimension:

THEOREM 1.10.

$$
\operatorname{dim}(\mathcal{M}(X): \mathbb{C}) \leq 4 \rho(X) \operatorname{dim}_{\mathbb{C}} X,
$$

where $\operatorname{dim}_{\mathbb{C}} X$ denotes the complex dimension of $X$.

This follows from Tsukamoto [17, Theorem 1.5].

EXAMPLE 1.11. If $X=\mathbb{C} P^{n}$, then we have

$$
\operatorname{dim}\left(\mathcal{M}\left(\mathbb{C} P^{n}\right): \mathbb{C}\right) \leq 4 \rho\left(\mathbb{C} P^{n}\right) n<4 n .
$$

Remark 1.12. M. Gromov gives a certain upper bound for dim $\left(\mathcal{M}\left(\mathbb{C} P^{n}\right): \mathbb{C}\right)$ in $[7$, p. 396, (c)]. Unfortunately, I could not find the definition of the Fubini-Study metric used in [7, p. 396, (c)] (the Fubini-Study metric has several conventions). Therefore I could not decide whether our estimate (6) is better than Gromov's estimate in [7, p. 396, (c)] or not. But Gromov referred to the paper of A. Eremenko [6] there, and the argument in [17, Lemma 2.1] is similar to the argument in [6, Theorem 2.5]. And I think that the use of packing density (or "mean energy" in [17]) makes the related estimates sharper.

Remark 1.13. Actually we mainly study "mean energy" instead of packing density in [17]. But many results for mean energy can be easily reformulated into the results for packing density.

\subsection{Organization of the paper}

In Section 2, we develop general theory of packing density and prove Theorem 1.6. We prove Theorem 1.3 in Section 3, Theorem 1.5 in Section 4 and Theorem 1.4 in Section 5. We prove Theorem 1.7 in Section 6 by using the Nevanlinna theory. Section 5 is independent of Section 3 and 4 . Section 6 is logically independent of all other sections. (But its meaning in the packing problem is underpinned by other results.) 


\section{§2. General theory}

In this section we study general properties of packing density. We discuss its scaling invariance in Subsection 2.1. We study upper bounds for packing density in Subsection 2.2, and we study lower bounds in Subsection 2.3. In this section $X$ is a Hermitian manifold, and $\omega$ denotes its Kähler form.

\subsection{Scaling invariance}

Let $f: \mathbb{C} \rightarrow X$ be a holomorphic map. Suppose that there exists a positive number $m<\infty$ such that

$$
|d f|(z) \leq m \text { for all } z \in \mathbb{C} .
$$

We define the holomorphic map $\hat{f}: \mathbb{C} \rightarrow X$ by setting $\hat{f}(z):=f(z / m)$. Then

$$
|d \hat{f}|(z)=\frac{1}{m}|d f|(z / m) \leq 1 .
$$

Hence $\hat{f} \in \mathcal{M}(X, \omega)$. The following can be easily checked:

Lemma 2.1.

$$
\limsup _{R \rightarrow \infty} \frac{1}{m^{2} \pi R^{2}} \int_{|z| \leq R}|d f|^{2} d x d y=\rho(\hat{f}) .
$$

Then we have the following scaling invariance:

Proposition 2.2. For any positive number $c$, we have

$$
\rho(X, c \omega)=\rho(X, \omega) .
$$

Here $\rho(X, c \omega)$ is the packing density of $X$ defined by using $c \omega$ as the metric form on $X$.

\subsection{Upper bounds for packing density}

To begin with, we introduce a key notion. For a positive number $\varepsilon>0$, let $\Delta(\varepsilon)$ be the disk of radius $\varepsilon$ in the plane:

$$
\Delta(\varepsilon):=\{z \in \mathbb{C}|| z \mid<\varepsilon\} .
$$

We use the natural Euclidean metric as the metric on $\Delta(\varepsilon)$. (So $\Delta(\varepsilon)$ is not the Poincaré disk.) 
Definition 2.3. ${ }^{1}$ A Hermitian manifold $X$ is $\varepsilon$-WFL (without flat lines) if there is no holomorphic isometric immersion from $\Delta(\varepsilon)$ to $X . X$ is WFL if $X$ is $\varepsilon$-WFL for all positive numbers $\varepsilon$. Here a holomorphic isometric immersion from $\Delta(\varepsilon)$ to $X$ is a holomorphic map $f: \Delta(\varepsilon) \rightarrow X$ satisfying $|d f|(z)=1$ for all $z \in \Delta(\varepsilon)$.

EXAmple 2.4. The complex projective line $\mathbb{C} P^{1}$ is WFL. (Actually all $\mathbb{C} P^{n}$ are also WFL; see Section 3.)

Proof. Suppose that there exists a holomorphic isometric immersion from $\Delta(\varepsilon)$ to $\mathbb{C} P^{1}$ for some positive number $\varepsilon$. This means that $\Delta(\varepsilon)$ is locally isometric to $\mathbb{C} P^{1}$. But $\Delta(\varepsilon)$ is flat and $\mathbb{C} P^{1}$ has a positive constant curvature. Hence it is impossible.

The following is the main result of this subsection.

TheOREM 2.5. Let $X$ be a compact Hermitian manifold and suppose $X$ is $\varepsilon$-WFL for some positive number $\varepsilon$. Then

$$
\rho(X)<1
$$

Note that we can suppose $X$ is $1 / 2$-WFL without loss of generality by using a scale change (cf. Proposition 2.2) if $X$ is $\varepsilon$-WFL for a positive number $\varepsilon$. The key for the proof of Theorem 2.5 is the following.

Proposition 2.6. Let $X$ be a $1 / 2-W F L$ compact Hermitian manifold and $K$ be a unit square in the complex plane $\mathbb{C}$. Then there exists a constant $c(K)<1$ such that

$$
\int_{K}|d f|^{2} d x d y \leq c(K) \quad \text { for all } f \in \mathcal{M}(X) .
$$

Proof. Since $\operatorname{vol}(K)=1$ and $|d f| \leq 1$, it is trivial that $\int_{K}|d f|^{2} d x d y \leq$ 1. Hence the proposition states that this trivial estimate can be improved.

Suppose the proposition is false. Then we have a sequence $\left\{f_{n}\right\}_{n \geq 1}$ in $\mathcal{M}(X)$ such that

$$
\int_{K}\left|d f_{n}\right|^{2} d x d y \rightarrow 1 \quad(n \rightarrow \infty)
$$

\footnotetext{
${ }^{1}$ The word "WFL" is inspired by the word "WFF" in Donaldson-Kronheimer [5, Definition (3.2.2)].
} 
Because $\left|d f_{n}\right| \leq 1$ and $X$ is compact, we can apply Arzela-Ascoli's theorem, and get a continuous map $f: \mathbb{C} \rightarrow X$ such that an appropriate subsequence of $\left\{f_{n}\right\}_{n \geq 1}$ converges to $f$ in the sense of uniform convergence on compact sets. Since each $f_{n}$ is holomorphic, $f$ is also holomorphic and

$$
\left|d f_{n}\right| \rightarrow|d f| \quad(n \rightarrow \infty) \quad \text { (uniform convergence on compact sets). }
$$

From $\left|d f_{n}\right| \leq 1$, we get $|d f| \leq 1$. From the assumption (7),

$$
\int_{K}|d f|^{2} d x d y=1 .
$$

Since $|d f| \leq 1$, this means

$$
|d f|(z)=1 \quad \text { for all } z \in K
$$

Then $f$ is a holomorphic isometric immersion from $K$ to $X$. This contradicts the assumption that $X$ is $1 / 2$-WFL (note that a unit square contains a open disk of radius $1 / 2$ ).

The above proposition states that there exists a constant $c(K)<1$ satisfying the statement for each unit square $K$. But we have the Euclidean symmetry. Hence we can suppose that

$c(K)=c\left(K^{\prime}\right)=: c<1 \quad$ for all unit squares $K$ and $K^{\prime}$ in the plane.

Proof of Theorem 2.5. As it is noted before, we can suppose that $X$ is $1 / 2$-WFL by using a scale change (this is just for simplicity). Let $f$ be an element of $\mathcal{M}(X)$ and $R$ be a positive number greater than $\sqrt{2}$. We prove $\rho(f) \leq c$ by packing unit squares in the disk $\bar{\Delta}(R)=\{z \in \mathbb{C}|| z \mid \leq$ $R\}$ (here we promise that unit square means "closed" unit square). Since the diameter of a unit square is $\sqrt{2}$, we have the following fact: If a unit square $K$ has a intersection with the disk $\bar{\Delta}(R-\sqrt{2}), K$ is contained in $\bar{\Delta}(R)$. Hence if we consider a tiling of the plane by unit squares, the disk $\bar{\Delta}(R-\sqrt{2})$ is covered by unit squares contained in $\bar{\Delta}(R)$. (See Figure 1 . The shaded area shows the unit squares which have intersections with the disk $\bar{\Delta}(R-\sqrt{2})$.) In other words, we have the following situation: There are unit squares $K_{1}, K_{2}, \ldots, K_{N}$ contained in $\bar{\Delta}(R)$ such that $K_{i}$ and $K_{j}$ $(i \neq j)$ have common points at most on their boundaries and

$$
\bar{\Delta}(R-\sqrt{2}) \subset \bigcup_{i=1}^{N} K_{i} \subset \bar{\Delta}(R) .
$$




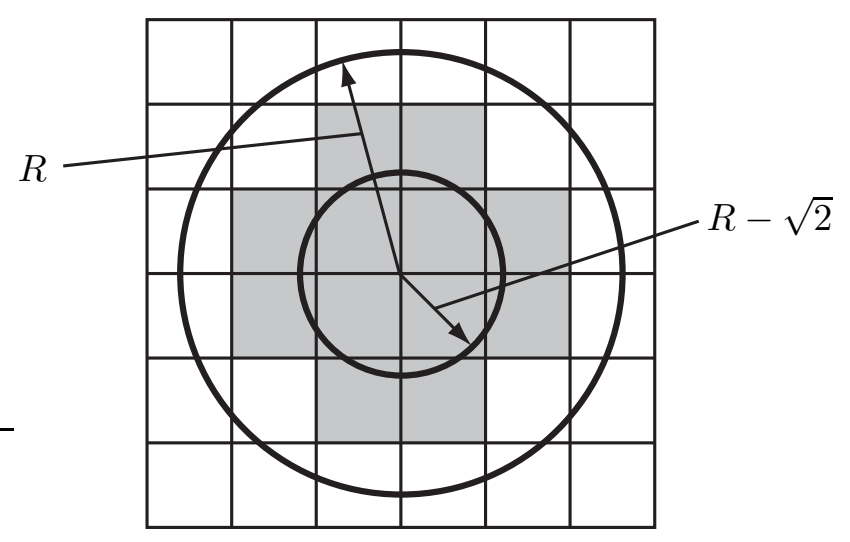

Figure 1: A tiling by unit squares.

Then $N=\operatorname{vol}\left(\bigcup K_{i}\right)$ satisfies

$$
\pi(R-\sqrt{2})^{2} \leq N \leq \pi R^{2}
$$

Therefore

$$
\begin{aligned}
\int_{\bar{\Delta}(R)}|d f|^{2} d x d y & =\sum_{i=1}^{N} \int_{K_{i}}|d f|^{2} d x d y+\int_{\bar{\Delta}(R) \backslash \cup K i}|d f|^{2} d x d y \\
& \leq N \cdot c+\left(\pi R^{2}-N\right) \\
& \leq \pi R^{2} \cdot c+\left\{\pi R^{2}-\pi(R-\sqrt{2})^{2}\right\}
\end{aligned}
$$

Here we have used Proposition 2.6 and $|d f| \leq 1$. Therefore

$$
\frac{1}{\pi R^{2}} \int_{\bar{\Delta}(R)}|d f|^{2} d x d y \leq c+\left\{1-\left(1-\frac{\sqrt{2}}{R}\right)^{2}\right\} .
$$

Taking the superior limit, we get

$$
\rho(f)=\limsup _{R \rightarrow \infty} \frac{1}{\pi R^{2}} \int_{\bar{\Delta}(R)}|d f|^{2} d x d y \leq c<1 .
$$

Thus

$$
\rho(X) \leq c<1
$$

Remark 2.7. The above argument does not give an effective estimate of the constant $c$. Hence we need another method if we want an explicit upper bound for $\rho(X)$. This is the theme of Section 5, and we prove Theorem 1.4 there. 


\subsection{Lower bounds for packing density}

Next we will establish lower bounds for $\rho(X)$ by using holomorphic maps from elliptic curves to $X$. Let $\mathbb{C} / \Lambda$ be an elliptic curve. Here $\Lambda$ denotes a lattice in the complex plane $\mathbb{C}$. The elliptic curve $\mathbb{C} / \Lambda$ has the Euclidean metric induced by the universal covering $\mathbb{C}$.

For a holomorphic map $f: \mathbb{C} / \Lambda \rightarrow X$, we define its energy $E(f)$ by setting

$$
E(f):=\int_{\mathbb{C} / \Lambda} f^{*} \omega=\int_{\mathbb{C} / \Lambda}|d f|^{2} d x d y .
$$

When $(X, \omega)=\left(\mathbb{C} P^{n}, \omega_{F S}\right)$, this is the degree of $f$ defined in (3). Let $\tilde{f}: \mathbb{C} \rightarrow X$ be the lift of $f$ and set $m:=\sup _{z \in \mathbb{C} / \Lambda}|d f|(z)$.

Proposition 2.8 .

$$
\lim _{R \rightarrow \infty} \frac{1}{\pi R^{2}} \int_{|z| \leq R}|d \tilde{f}|^{2} d x d y=\frac{E(f)}{\operatorname{vol}(\mathbb{C} / \Lambda)} .
$$

Proof. We can prove this proposition by packing the fundamental domains of $\Lambda \subset \mathbb{C}$ in the plane. The argument is similar to the proof of Theorem 2.5 and we omit the detail.

THEOREM 2.9. If there exists a non-constant holomorphic map $f$ : $\mathbb{C} / \Lambda \rightarrow X$, then we have the following estimate.

$$
\rho(X) \geq \frac{E(f)}{\|d f\|_{\infty}^{2} \operatorname{vol}(\mathbb{C} / \Lambda)} .
$$

In particular,

$$
\rho(X)>0 .
$$

Proof. Note that $m=\|d f\|_{\infty}$ and $E(f)$ are positive because $f$ is a non-constant map. From Lemma 2.1 and Proposition 2.8,

$$
0<\frac{E(f)}{\|d f\|_{\infty}^{2} \operatorname{vol}(\mathbb{C} / \Lambda)}=\lim _{R \rightarrow \infty} \frac{1}{m^{2} \pi R^{2}} \int_{|z| \leq R}|d \tilde{f}|^{2} d x d y \leq \rho(X) .
$$

As a corollary of Theorem 2.9, we get Theorem 1.6:

Proof of Theorem 1.6. If $f: \mathbb{C} / \Lambda \rightarrow \mathbb{C} P^{n}$ is a constant map, then the statement is trivial. When $f$ is a non-constant map, the statement follows from Theorem 2.9 (here we have $E(f)=\operatorname{deg}(f)$.) 


\section{§3. Proof of Theorem 1.3}

We give the proof of Theorem 1.3 in this section. Most of the proof have already been done in Section 2. The last piece which we need is the following.

\section{Proposition 3.1. The complex projective space $\mathbb{C} P^{n}$ is $W F L$.}

This follows from a general result of E. Calabi concerning isometric imbeddings of complex manifolds [3, Theorem 8]. This proposition is one of the most fundamental result for the theory of "packing problem for holomorphic curves". So, for the completion of the argument, we will give its proof (which is not essentially different from the argument of [3]). Recall the following easy fact:

LEMMA 3.2. Let $f\left(z_{1}, z_{2}\right)$ be a holomorphic function in two variables defined on a connected open neighborhood of the origin in $\mathbb{C}^{2}$. Set $g(z):=$ $f(z, \bar{z}) . g(z)$ is defined on a neighborhood of the origin in $\mathbb{C}$. If $g(z) \equiv 0$, then we have $f\left(z_{1}, z_{2}\right) \equiv 0$.

Proof. Differentiating the equation $g(z)=f(z, \bar{z})$, we have

$$
\begin{aligned}
\frac{\partial}{\partial z} g(z) & :=\frac{1}{2}\left(\frac{\partial}{\partial x}-\sqrt{-1} \frac{\partial}{\partial y}\right) g(z)=\frac{\partial f}{\partial z_{1}}(z, \bar{z}), \\
\frac{\partial}{\partial \bar{z}} g(z) & :=\frac{1}{2}\left(\frac{\partial}{\partial x}+\sqrt{-1} \frac{\partial}{\partial y}\right) g(z)=\frac{\partial f}{\partial z_{2}}(z, \bar{z}) .
\end{aligned}
$$

More generally we have

$$
\frac{\partial^{n+m}}{\partial z^{n} \partial \bar{z}^{m}} g(z)=\frac{\partial^{n+m} f}{\partial z_{1}^{n} \partial z_{2}^{m}}(z, \bar{z}) \quad \text { for all } n, m \geq 0 .
$$

Hence, if $g(z) \equiv 0$, then all partial derivatives of $f$ at $\left(z_{1}, z_{2}\right)=(0,0)$ are zero. This means that $f\left(z_{1}, z_{2}\right) \equiv 0$.

Proof of Proposition 3.1. ${ }^{2}$ Suppose that there exists a holomorphic isometric immersion $f: \Delta(\varepsilon) \rightarrow \mathbb{C} P^{n}$ for some positive number $\varepsilon$. We can

\footnotetext{
${ }^{2}$ This proof implicitly uses the notions "diastasis" and "resolvability of rank $n$ " in Calabi [3, p. 3 and p. 11]. So this argument is essentially contained in the proof of $[3$, Theorem 8].
} 
suppose that $f(0)=[1: 0: \cdots: 0]$ without loss of generality. Then we can express $f$ in some neighborhood of the origin by

$$
f(z)=\left[1: f_{1}(z): f_{2}(z): \cdots: f_{n}(z)\right]
$$

where $f_{i}(z)$ is a holomorphic function such that $f_{i}(0)=0(1 \leq i \leq n)$.

The following argument is purely local. Hence we promise that all functions are defined on some neighborhood of the origin in the complex plane $\mathbb{C}$. From the definition of the Fubini-Study metric (1), we have

$$
|d f|^{2}(z)=\frac{1}{4 \pi} \Delta \log \left(1+\sum_{i=1}^{n}\left|f_{i}(z)\right|^{2}\right) \quad\left(\Delta:=\frac{\partial^{2}}{\partial x^{2}}+\frac{\partial^{2}}{\partial y^{2}}\right) .
$$

Since $f$ is a holomorphic isometric immersion, $|d f|^{2} \equiv 1$. On the other hand, we have

$$
\frac{1}{4 \pi} \Delta\left(\pi|z|^{2}\right) \equiv 1
$$

Hence

$$
\Delta\left\{\log \left(1+\sum\left|f_{i}(z)\right|^{2}\right)-\pi|z|^{2}\right\} \equiv 0
$$

Because a harmonic function is locally the real part of a holomorphic function, we have a holomorphic function $g(z)$ such that $g(0)=0$ and

$$
\log \left(1+\sum\left|f_{i}(z)\right|^{2}\right)=\pi|z|^{2}+g(z)+\overline{g(z)}
$$

Introducing the new holomorphic functions $\bar{f}_{i}(z):=\overline{f_{i}(\bar{z})}$ and $\bar{g}(z):=\overline{g(\bar{z})}$, we can express the above equation by

$$
\log \left(1+\sum f_{i}(z) \bar{f}_{i}(\bar{z})\right)=\pi z \bar{z}+g(z)+\bar{g}(\bar{z}) .
$$

Applying Lemma 3.2 to this, we get

$$
\log \left(1+\sum f_{i}\left(z_{1}\right) \bar{f}_{i}\left(z_{2}\right)\right)=\pi z_{1} z_{2}+g\left(z_{1}\right)+\bar{g}\left(z_{2}\right)
$$

Substituting $z_{2}=0$, we get $g\left(z_{1}\right) \equiv 0$ because $\bar{f}_{i}(0)=\bar{g}(0)=0$. Thus the above equation becomes

$$
\log \left(1+\sum f_{i}\left(z_{1}\right) \bar{f}_{i}\left(z_{2}\right)\right)=\pi z_{1} z_{2} .
$$

Hence we have

$$
1+\sum_{i=1}^{n} f_{i}\left(z_{1}\right) \bar{f}_{i}\left(z_{2}\right)=\exp \left(\pi z_{1} z_{2}\right)
$$


Applying $\partial^{\alpha+\beta} / \partial z_{1}^{\alpha} \partial z_{2}^{\beta}$ to this at $\left(z_{1}, z_{2}\right)=(0,0)$, we get

$$
\sum_{i=1}^{n} f_{i}^{(\alpha)}(0) \overline{f_{i}^{(\beta)}(0)}=\pi^{\alpha} \alpha ! \delta_{\alpha \beta} \quad \text { for } \alpha, \beta \geq 1 \text {. }
$$

Here $f_{i}^{(\alpha)}(0)$ is the $\alpha$-th derivative of $f_{i}$ at the origin and $\delta_{\alpha \beta}$ is the Kronecker delta. This means that an infinite number of non-zero vectors $\left(f_{1}^{(\alpha)}(0), f_{2}^{(\alpha)}(0), \ldots, f_{n}^{(\alpha)}(0)\right)(\alpha \geq 1)$ in $\mathbb{C}^{n}$ are orthogonal to each other. It is impossible.

Proof of Theorem 1.3. The complex projective space $\mathbb{C} P^{n}$ satisfies all the conditions required in Theorem 2.5. Hence we get

$$
\rho\left(\mathbb{C} P^{n}\right)<1 .
$$

On the other hand there are non-constant elliptic functions; for example, Weierstrass' elliptic function $\wp(z)$. Then we can apply Theorem 2.9, and we get

$$
\rho\left(\mathbb{C} P^{1}\right)>0 .
$$

Since the natural inclusion $\mathbb{C} P^{1} \hookrightarrow \mathbb{C} P^{n}$ is a holomorphic isometric imbedding, we conclude that

$$
\rho\left(\mathbb{C} P^{n}\right) \geq \rho\left(\mathbb{C} P^{1}\right)>0 .
$$

\section{§4. Packing density and theta functions}

We prove Theorem 1.5 in this section. The proof is based on the lower bound given in Theorem 2.9. In order to apply it, we need holomorphic maps from elliptic curves to the projective space. The classical theory of theta functions (or the theorem of Tian [15]) gives a good answer. Let $\tau=s+t \sqrt{-1}$ be an element of the upper half plane $(t=\operatorname{Im} \tau>0)$. We define the theta function $\theta(z)$ by setting

$$
\theta(z):=\sum_{n \in \mathbb{Z}} \exp \left(\pi \sqrt{-1} n^{2} \tau+2 \pi \sqrt{-1} n z\right) \quad \text { for } z \in \mathbb{C} .
$$

We define the theta function $\theta_{a, b}(z)$ with characteristics $a, b \in \mathbb{R}$ by setting

$$
\theta_{a, b}(z):=\exp \left(\pi \sqrt{-1} a^{2} \tau+2 \pi \sqrt{-1} a(z+b)\right) \theta(z+a \tau+b) .
$$


We can construct projective imbeddings of elliptic curves by using these $\theta_{a, b}(z)$. We follow the arguments in Mumford [13, Chapter 1, §4].

We define a lattice $\Lambda$ in $\mathbb{C}$ by setting $\Lambda:=\mathbb{Z} \oplus \mathbb{Z} \tau$. For any integer $l \geq 2$, we set

$$
\begin{gathered}
\left(\frac{1}{l} \mathbb{Z}\right)^{2} \cap[0,1)^{2}=\left\{\left(a_{0}, b_{0}\right),\left(a_{1}, b_{1}\right), \ldots,\left(a_{l^{2}-1}, b_{l^{2}-1}\right)\right\}, \\
\theta_{i}(z):=\theta_{a_{i}, b_{i}}(z) \quad\left(0 \leq i \leq l^{2}-1\right), \\
\varphi_{l}: \mathbb{C} / l \Lambda \longrightarrow\left[\theta_{0}(z): \theta_{1}(z): \cdots: \theta_{l^{2}-1}(z)\right] .
\end{gathered}
$$

It is well-known that $\varphi_{l}$ becomes a holomorphic imbedding and $\operatorname{deg}\left(\varphi_{l}\right)=l^{2}$ (see $[13$, Chapter $1, \S 4])$. Since $\operatorname{vol}(\mathbb{C} / l \Lambda)=t l^{2}(t=\operatorname{Im} \tau)$, Theorem 2.9 gives the following lower bound:

$$
\rho\left(\mathbb{C} P^{l^{2}-1}\right) \geq \frac{1}{t\left\|d \varphi_{l}\right\|_{\infty}^{2}} .
$$

Therefore we need the estimate of $\left\|d \varphi_{l}\right\|_{\infty}$.

Proposition 4.1.

$$
\lim _{l \rightarrow \infty}\left\|\left|d \varphi_{l}\right|^{2}-\frac{1}{t}\right\|_{\infty}=0
$$

Hence we have

$$
\lim _{l \rightarrow \infty}\left\|d \varphi_{l}\right\|_{\infty}^{2}=\frac{1}{t}
$$

This can be considered as a special case of the result of Tian [15]. (See also Ji [8], Zelditch [18] and the references therein.) But for the completion of the argument, we will give its direct proof below. I think that probably the paper of Kempf [9] essentially contains the direct proof of this result although I did not get this paper.

LEMMA 4.2 .

$$
\int_{0}^{1} \int_{0}^{1}\left|\theta_{a, b}(z)\right|^{2} d a d b=\sqrt{\frac{1}{2 t}} \exp \left(\frac{2 \pi y^{2}}{t}\right) .
$$

Hence we have

$$
\frac{1}{4 \pi} \Delta \log \int_{0}^{1} \int_{0}^{1}\left|\theta_{a, b}(z)\right|^{2} d a d b \equiv \frac{1}{t}, \quad\left(\Delta=\frac{\partial^{2}}{\partial x^{2}}+\frac{\partial^{2}}{\partial y^{2}}\right) .
$$


Proof. The proof is just a calculation.

$$
\begin{gathered}
\left|\theta_{a, b}(z)\right|=\exp \left(-\pi a^{2} t-2 \pi a y\right)|\theta(z+a \tau+b)|, \\
\theta(z+a \tau+b)=\sum_{n} \exp \left(\pi \sqrt{-1} n^{2} \tau+2 \pi \sqrt{-1} n(z+a \tau)\right) \exp (2 \pi \sqrt{-1} n b) .
\end{gathered}
$$

From Parseval's equality

$$
\begin{aligned}
\int_{0}^{1}|\theta(z+a \tau+b)|^{2} d b & =\sum_{n}\left|\exp \left(\pi \sqrt{-1} n^{2} \tau+2 \pi \sqrt{-1} n(z+a \tau)\right)\right|^{2} \\
& =\sum_{n} \exp \left(-2 \pi n^{2} t-4 \pi n(y+a t)\right) .
\end{aligned}
$$

Hence

$$
\begin{aligned}
\int_{0}^{1}\left|\theta_{a, b}(z)\right|^{2} d b & =\exp \left(-2 \pi a^{2} t-4 \pi a y\right) \sum_{n} \exp \left(-2 \pi n^{2} t-4 \pi n(y+a t)\right) \\
& =\sum_{n} \exp \left(-2 \pi t(a+n)^{2}-4 \pi y(a+n)\right) .
\end{aligned}
$$

Thus we get

$$
\begin{aligned}
\int_{0}^{1} d a \int_{0}^{1}\left|\theta_{a, b}(z)\right|^{2} d b & =\sum_{n} \int_{0}^{1} \exp \left(-2 \pi t(a+n)^{2}-4 \pi y(a+n)\right) d a \\
& =\sum_{n} \int_{n}^{n+1} \exp \left(-2 \pi t a^{2}-4 \pi y a\right) d a \\
& =\int_{-\infty}^{+\infty} \exp \left(-2 \pi t a^{2}-4 \pi y a\right) d a \\
& =\sqrt{\frac{1}{2 t}} \exp \left(\frac{2 \pi y^{2}}{t}\right) .
\end{aligned}
$$

Lemma 4.3. For $\alpha, \beta \in \mathbb{Z}$, we have

$$
\left|d \varphi_{l}\right|(z+\alpha \tau+\beta)=\left|d \varphi_{l}\right|(z) .
$$

In other words, $\left|d \varphi_{l}\right|(z)$ is invariant under the following $\mathbb{Z}^{2}$-action on $\mathbb{C} / l \Lambda$.

$$
\mathbb{Z}^{2} \curvearrowright \mathbb{C} / l \Lambda, \quad((\alpha, \beta), z) \longmapsto z+\alpha \tau+\beta .
$$

(Actually this result is still true for $\alpha, \beta \in \frac{1}{l} \mathbb{Z}$. But we don't need it.) 
Proof.

$$
\begin{aligned}
& \theta_{a, b}(z+\alpha \tau+\beta) \\
& \quad=\exp \left(-\pi \sqrt{-1} \alpha^{2} \tau-2 \pi \sqrt{-1} \alpha z\right) \exp (-2 \pi \sqrt{-1} \alpha b) \theta_{a+\alpha, b+\beta}(z) .
\end{aligned}
$$

Since $\alpha$ and $\beta$ are integers, we have

$$
\theta_{a+\alpha, b+\beta}(z)=\exp (2 \pi \sqrt{-1} a \beta) \theta_{a, b}(z) .
$$

Set $c_{i}:=\exp \left(-2 \pi \alpha b_{i}+2 \pi \sqrt{-1} a_{i} \beta\right) \in U(1),\left(0 \leq i \leq l^{2}-1\right)$. Then

$$
\theta_{i}(z+\alpha \tau+\beta)=\exp \left(-\pi \sqrt{-1} \alpha^{2} \tau-2 \pi \sqrt{-1} \alpha z\right) c_{i} \theta_{i}(z) .
$$

Hence (this is the "equivariance" described in [13, Chapter 1, §4])

$$
\varphi_{l}(z+\alpha \tau+\beta)=\left[c_{0} \theta_{0}(z): c_{1} \theta_{1}(z): \cdots: c_{l^{2}-1} \theta_{l^{2}-1}(z)\right] .
$$

Therefore

$$
\begin{aligned}
\left|d \varphi_{l}\right|^{2}(z+\alpha \tau+\beta) & =\frac{1}{4 \pi} \Delta \log \sum_{i}\left|c_{i} \theta_{i}(z)\right|^{2} \\
& =\frac{1}{4 \pi} \Delta \log \sum_{i}\left|\theta_{i}(z)\right|^{2} \\
& =\left|d \varphi_{l}\right|^{2}(z) .
\end{aligned}
$$

Proof of Proposition 4.1. From the definition of the Fubini-Study met$\operatorname{ric}(1)$,

$$
\left|d \varphi_{l}\right|^{2}(z)=\frac{1}{4 \pi} \Delta \log \sum_{i}\left|\theta_{i}(z)\right|^{2}=\frac{1}{4 \pi} \Delta \log \left(\frac{1}{l^{2}} \sum_{i}\left|\theta_{i}(z)\right|^{2}\right) .
$$

Since $[0,1)^{2}=\bigsqcup_{i}\left[a_{i}, a_{i}+1 / l\right) \times\left[b_{i}, b_{i}+1 / l\right)$ is a division into small squares, the definition of the Riemann integral gives the following point-wise convergence.

$$
\begin{aligned}
\lim _{l \rightarrow \infty} \frac{1}{l^{2}} \sum_{i}\left|\theta_{i}(z)\right|^{2} & =\int_{0}^{1} \int_{0}^{1}\left|\theta_{a, b}(z)\right|^{2} d a d b \\
& =\sqrt{\frac{1}{2 t}} \exp \left(\frac{2 \pi y^{2}}{t}\right) \text { for any } z \in \mathbb{C} .
\end{aligned}
$$


Actually we can say more. Set $K:=\{x+y \tau \in \mathbb{C} \mid 0 \leq x, y \leq 1\}$. Since $K$ is compact, it is easy to see that

$$
\lim _{l \rightarrow \infty}\left\|\frac{1}{l^{2}} \sum_{i}\left|\theta_{i}(z)\right|^{2}-\int_{0}^{1} \int_{0}^{1}\left|\theta_{a, b}(z)\right|^{2} d a d b\right\|_{\mathcal{C}^{k}(K)}=0 \quad \text { for all } k \geq 0 .
$$

Here $\|\cdot\|_{\mathcal{C}^{k}(K)}$ is the $\mathcal{C}^{k}$-norm for functions defined over $K$. Therefore we have

$$
\lim _{l \rightarrow \infty}\left\|\frac{1}{4 \pi} \Delta \log \left(\frac{1}{l^{2}} \sum_{i}\left|\theta_{i}(z)\right|^{2}\right)-\frac{1}{4 \pi} \Delta \log \int_{0}^{1} \int_{0}^{1}\left|\theta_{a, b}(z)\right|^{2} d a d b\right\|_{\mathcal{C}^{0}(K)}=0 .
$$

Hence

$$
\lim _{l \rightarrow \infty}\left\|\left|d \varphi_{l}\right|^{2}-\frac{1}{t}\right\|_{\mathcal{C}^{0}(K)}=0 .
$$

Here we consider $K$ as a subspace of the elliptic curve $\mathbb{C} / l \Lambda$ through the natural projection $\mathbb{C} \rightarrow \mathbb{C} / l \Lambda$. Since $K$ is a fundamental domain of the $\mathbb{Z}^{2}$-symmetry described in Lemma 4.3 , we get the conclusion:

$$
\lim _{l \rightarrow \infty}\left\|\left|d \varphi_{l}\right|^{2}-\frac{1}{t}\right\|_{\mathcal{C}^{0}(\mathbb{C} / l \Lambda)}=0
$$

Proof of Theorem 1.5. From the inequality (9) and Proposition 4.1, we have

$$
\liminf _{l \rightarrow \infty} \rho\left(\mathbb{C} P^{l^{2}-1}\right) \geq \lim _{l \rightarrow \infty} \frac{1}{t\left\|d \varphi_{l}\right\|_{\infty}^{2}}=1 .
$$

Hence $\lim _{l \rightarrow \infty} \rho\left(\mathbb{C} P^{l^{2}-1}\right)=1$. Since $\rho\left(\mathbb{C} P^{n}\right)$ is monotone increasing in $n$, we get

$$
\lim _{n \rightarrow \infty} \rho\left(\mathbb{C} P^{n}\right)=1
$$

\section{§5. Explicit upper bounds for $\rho\left(\mathbb{C} P^{1}\right)$}

In this section we prove Theorem 1.4. To simplify the calculations, we will use the following rescaled Fubini-Study metric $d s^{2}$ as the metric on $\mathbb{C} P^{1}$ 
in this section (cf. Proposition 2.2).

$$
d s^{2}:=\frac{d w d \bar{w}}{\left(1+|w|^{2}\right)^{2}}
$$

the fundamental 2-form of $d s^{2}=\frac{\sqrt{-1}}{2} \frac{d w \wedge d \bar{w}}{\left(1+|w|^{2}\right)^{2}} \quad\left(=\pi \omega_{F S}\right), \quad($ cf. (1)).

Here $w$ is the natural coordinate on $\mathbb{C}$ of $\mathbb{C} P^{1}=\mathbb{C} \cup\{\infty\}$. Then for a meromorphic function $f(z)$ in the complex plane, the norm of the differential $d f$ is given by

$$
|d f|(z)=\frac{\left|f^{\prime}(z)\right|}{1+|f(z)|^{2}}
$$

\subsection{Preliminary estimates}

In this subsection we prepare various estimates for the proof of Theorem 1.4. Here we will not pursue precise estimates. Actually we will use many loose estimates for simplicity of the calculations. Our purpose is to show the fact that we can get an explicit upper bound on packing densities.

To begin with, we compute the distance on $\mathbb{C} P^{1}$ defined by the metric (10).

Lemma 5.1. Let $w$ be a point of $\mathbb{C} P^{1}$. Then we have

$$
d_{\mathbb{C} P^{1}}(0, w)=\arctan |w| .
$$

Here $d_{\mathbb{C} P^{1}}(\cdot, \cdot)$ is the distance on $\mathbb{C} P^{1}$ defined by $(10)$, and $\arctan (\cdot)$ is the branch of the inverse function of $\tan (\cdot)$ satisfying $\arctan 0=0$.

Proof. $c(t):=w t(0 \leq t \leq 1)$ is the minimum geodesic from 0 to $w$. Hence we get

$$
d_{\mathbb{C} P^{1}}(0, w)=\int_{0}^{1} \frac{\left|c^{\prime}(t)\right|}{1+|c(t)|^{2}} d t=\int_{0}^{1} \frac{|w|}{1+|w|^{2} t^{2}} d t=\arctan |w| .
$$

We set $r:=|z|$ for $z$ in the complex plane and put

$$
\varepsilon:=10^{-100} \text { and } r_{0}:=10^{-10} \text {. }
$$

Let $f(z)$ be a meromorphic function in the complex plane which satisfies

$$
|d f|(z)=\frac{\left|f^{\prime}(z)\right|}{1+|f(z)|^{2}} \leq 1 \quad \text { for all } z \in \mathbb{C} .
$$


In addition, we suppose that the following conditions are also satisfied:

$$
f(0)=0 \quad \text { and } \quad 1-\varepsilon \leq|d f|(0) \leq 1 .
$$

The main purpose of this subsection is to get a good estimate of $|d f|^{2}(z)$ in the disk $r \leq r_{0}$ under the conditions (11) and (12). The following lemma is the basis of our argument.

LEMMA 5.2.

$$
|f(z)|^{2} \leq \tan ^{2} r \leq r^{2}+2 r^{4} \leq 2 r^{2} \quad\left(r \leq r_{0}\right) .
$$

Let $f(z)=a_{1} z+a_{2} z^{2}+a_{3} z^{3}+\cdots$ be the Taylor expansion centered at the origin. Then we have

$$
1-\varepsilon \leq\left|a_{1}\right| \leq 1 \quad \text { and } \quad\left|a_{n}\right| \leq(4 / \pi)^{n}<2^{n} .
$$

Proof. Since $|d f| \leq 1$ and $f(0)=0$, we have

$$
\arctan |f(z)|=d_{\mathbb{C} P^{1}}(f(0), f(z)) \leq r .
$$

Hence

$$
|f(z)| \leq \tan r \quad(r<\pi / 2)
$$

Here we have

$$
\tan ^{2} r=\frac{\sin ^{2} r}{1-\sin ^{2} r}=\sin ^{2} r+\frac{\sin ^{4} r}{1-\sin ^{2} r} \leq r^{2}+2 r^{4} \leq 2 r^{2} \quad\left(r \leq r_{0}\right) .
$$

Hence we get the above first statement. (Of course, this is a very loose estimate.)

Next we will estimate the coefficients of the Taylor expansion. Since $|d f|(0)=\left|f^{\prime}(0)\right|=\left|a_{1}\right|$, we have

$$
1-\varepsilon \leq\left|a_{1}\right| \leq 1
$$

Using (13) at $r=\pi / 4$, we have

$$
|f(z)| \leq 1 \quad(r=\pi / 4) .
$$

Thus we get

$$
\left|a_{n}\right|=\left|\frac{1}{2 \pi \sqrt{-1}} \int_{|z|=\pi / 4} \frac{f(z)}{z^{n+1}} d z\right| \leq(4 / \pi)^{n}<2^{n} .
$$


In the following estimates, we always assume $r \leq r_{0}$. The important term in the estimate of $|d f|^{2}$ is the second order term and the higher order terms will be loosely estimated. First we will estimate the denominator of $|d f|^{2}(z)=\left|f^{\prime}(z)\right|^{2} /\left(1+|f(z)|^{2}\right)^{2}$ :

LEMMA 5.3.

$$
\frac{1}{\left(1+|f(z)|^{2}\right)^{2}} \leq 1-2 r^{2}+30 r^{3}+\varepsilon \quad\left(r \leq r_{0}\right) .
$$

Proof. Using Lemma 5.2, if $r \leq r_{0}$, we have

$$
\begin{aligned}
\frac{1}{\left(1+|f(z)|^{2}\right)^{2}} & =1-2|f(z)|^{2}+\frac{3|f(z)|^{4}+2|f(z)|^{6}}{\left(1+|f(z)|^{2}\right)^{2}} \\
& \leq 1-2|f(z)|^{2}+3 \cdot 2^{2} \cdot r^{4}+2 \cdot 2^{3} \cdot r^{6} \\
& =1-2|f(z)|^{2}+\left(3 \cdot 2^{2}+2^{4} \cdot r^{2}\right) r^{4} \\
& \leq 1-2|f(z)|^{2}+13 r^{4}
\end{aligned}
$$

The term $-2|f(z)|^{2}$ can be estimated as follows.

$$
|f(z)| \geq\left|a_{1} z\right|-\left|a_{2} z^{2}+a_{3} z^{3}+\cdots\right| .
$$

And we have

$$
\left|a_{2} z^{2}+a_{3} z^{3}+\cdots\right| \leq 2^{2} \cdot r^{2}+2^{3} \cdot r^{3}+\cdots=\frac{4 r^{2}}{1-2 r} \leq 5 r^{2} .
$$

Hence

$$
|f(z)| \geq\left|a_{1}\right| r-5 r^{2} \geq(1-\varepsilon) r-5 r^{2} \geq 0
$$

Then

$$
|f(z)|^{2} \geq(1-\varepsilon)^{2} r^{2}-10(1-\varepsilon) r^{3}+25 r^{4} \geq(1-2 \varepsilon) r^{2}-10 r^{3} .
$$

Thus

$$
-2|f(z)|^{2} \leq-2(1-2 \varepsilon) r^{2}+20 r^{3} .
$$

Hence we get the conclusion:

$$
\begin{aligned}
\frac{1}{\left(1+|f(z)|^{2}\right)^{2}} & \leq 1-2(1-2 \varepsilon) r^{2}+20 r^{3}+13 r^{4} \\
& =1-2 r^{2}+(20+13 r) r^{3}+4 \varepsilon r^{2} \\
& \leq 1-2 r^{2}+30 r^{3}+\varepsilon .
\end{aligned}
$$


Next we will estimate the numerator $\left|f^{\prime}(z)\right|^{2}$. This case is more complicated and we need some preparations.

LEMMA 5.4.

$$
2\left|a_{2}\right| \leq 30 \sqrt{\varepsilon}
$$

Proof.

$$
f^{\prime}(z)=a_{1}+2 a_{2} z+3 a_{3} z^{2}+4 a_{4} z^{3}+\cdots .
$$

Using $|d f| \leq 1$ and $r \leq r_{0}$, we have

$$
\left|f^{\prime}(z)\right| \leq 1+|f(z)|^{2} \leq 1+2 r^{2}
$$

Hence

$$
\begin{aligned}
\left|a_{1}+2 a_{2} z\right| & \leq 1+2 r^{2}+\left|3 a_{3} z^{2}+4 a_{4} z^{3}+5 a_{5} z^{4}+\cdots\right| \\
& \leq 1+2 r^{2}+\left(3 \cdot 2^{3} \cdot r^{2}+4 \cdot 2^{4} \cdot r^{3}+5 \cdot 2^{5} \cdot r^{4}+\cdots\right) \\
& =1+2 r^{2}+\frac{24 r^{2}-32 r^{3}}{(1-2 r)^{2}} \\
& \leq 1+r^{2}\left(2+\frac{24}{(1-2 r)^{2}}\right) \\
& \leq 1+27 r^{2}
\end{aligned}
$$

Let $\theta_{n} \in \mathbb{R} / 2 \pi \mathbb{Z}$ be the argument of $a_{n}: a_{n}=\left|a_{n}\right| e^{\sqrt{-1} \theta_{n}}$. If $a_{n}=0$, then we set $\theta_{n}:=0$. Putting $z=\sqrt{\varepsilon} e^{\sqrt{-1}\left(\theta_{1}-\theta_{2}\right)}$ in the above, we get

$$
\left|a_{1}\right|+2\left|a_{2}\right| \sqrt{\varepsilon} \leq 1+27 \varepsilon .
$$

Since $\left|a_{1}\right| \geq 1-\varepsilon$,

$$
2\left|a_{2}\right| \leq \frac{\varepsilon+27 \varepsilon}{\sqrt{\varepsilon}} \leq 30 \sqrt{\varepsilon}
$$

Set $E(r):=4\left|a_{4}\right| r^{3}+5\left|a_{5}\right| r^{4}+6\left|a_{6}\right| r^{5}+\cdots$. ("E" is the initial letter of "error term".)

LEMMA 5.5.

$$
E(r) \leq 65 r^{3} \leq 10^{-20} \quad\left(r \leq r_{0}\right)
$$


Proof. Using Lemma 5.2 (and $r \leq r_{0}$ ), we have

$$
\begin{aligned}
E(r) & \leq 4 \cdot 2^{4} \cdot r^{3}+5 \cdot 2^{5} \cdot r^{4}+6 \cdot 2^{6} \cdot r^{5}+\cdots \\
& =\frac{64 r^{3}-96 r^{4}}{(1-2 r)^{2}} \leq \frac{64 r^{3}}{(1-2 r)^{2}} \leq 65 r^{3} .
\end{aligned}
$$

Since $r_{0}=10^{-10}$, we have $65 r^{3}<10^{-20}$.

Lemma 5.6. Set $\delta:=10^{-5}$. We have

$$
3\left|a_{3}\right| r^{2} \leq r^{2}+100 r^{3}+\delta \sqrt{\varepsilon} \quad\left(r \leq r_{0}\right) .
$$

Proof. From Lemma 5.2,

$$
\left|a_{1}+2 a_{2} z+3 a_{3} z^{2}+4 a_{4} z^{3}+\cdots\right|=\left|f^{\prime}(z)\right| \leq 1+|f(z)|^{2} \leq 1+r^{2}+2 r^{4} .
$$

Using Lemma 5.4 and Lemma 5.5, we have

$$
\begin{aligned}
\left|a_{1}+3 a_{3} z^{2}\right| & \leq 1+r^{2}+2 r^{4}+\left|2 a_{2} z+4 a_{4} z^{3}+5 a_{5} z^{4}+\cdots\right| \\
& \leq 1+r^{2}+2 r^{4}+30 \sqrt{\varepsilon} r+E(r) \\
& \leq 1+r^{2}+(2 r+65) r^{3}+30 \sqrt{\varepsilon} r \\
& \leq 1+r^{2}+100 r^{3}+30 \sqrt{\varepsilon} r .
\end{aligned}
$$

Let $\theta_{n}$ be the argument of $a_{n}$ as in the proof of Lemma 5.4. Putting $z=$ $r e^{\frac{\sqrt{-1}}{2}\left(\theta_{1}-\theta_{3}\right)}$ in the above, we get

$$
\left|a_{1}\right|+3\left|a_{3}\right| r^{2} \leq 1+r^{2}+100 r^{3}+30 \sqrt{\varepsilon} r .
$$

Since $\left|a_{1}\right| \geq 1-\varepsilon$, we have

$$
3\left|a_{3}\right| r^{2} \leq \varepsilon+r^{2}+100 r^{3}+30 \sqrt{\varepsilon} r \leq r^{2}+100 r^{3}+\delta \sqrt{\varepsilon} .
$$

Set

$$
\cos ^{+} x:=\max (0, \cos x) \text { for all } x \in \mathbb{R} .
$$

LEMMA 5.7.

$$
\left|f^{\prime}(z)\right|^{2} \leq 1+2 r^{2} \cos ^{+}\left(2 \theta-\theta_{1}+\theta_{3}\right)+500 r^{3}+\frac{1}{2} \sqrt{\varepsilon} \quad\left(r \leq r_{0}\right) .
$$

Here $\theta$ is the argument of $z$ and $\theta_{n}$ is the argument of $a_{n}$. 
Proof. Since $f^{\prime}(z)=a_{1}+2 a_{2} z+3 a_{3} z^{2}+\cdots$, we have

$$
\begin{aligned}
\left|f^{\prime}(z)\right| & \leq\left|a_{1}+3 a_{3} z^{2}\right|+\left|2 a_{2} z\right|+\left|4 a_{4} z^{3}+5 a_{5} z^{4}+6 a_{6} z^{5}+\cdots\right| \\
& \leq\left|a_{1}+3 a_{3} z^{2}\right|+30 \sqrt{\varepsilon} r+E(r) .
\end{aligned}
$$

Hence

$$
\begin{aligned}
\left|f^{\prime}(z)\right|^{2} \leq \mid & a_{1}+\left.3 a_{3} z^{2}\right|^{2}+E(r)^{2}+2\left|a_{1}+3 a_{3} z^{2}\right| \cdot E(r) \\
& +900 \varepsilon r^{2}+2\left|a_{1}+3 a_{3} z^{2}\right| \cdot 30 \sqrt{\varepsilon} r+60 \sqrt{\varepsilon} r \cdot E(r) .
\end{aligned}
$$

Since $2\left|a_{1}+3 a_{3} z^{2}\right| \leq 2\left|a_{1}\right|+6\left|a_{3}\right| r^{2} \leq 2+6 \cdot 2^{3} \cdot r^{2} \leq 3$,

$$
\begin{aligned}
900 & \varepsilon r^{2}+2\left|a_{1}+3 a_{3} z^{2}\right| \cdot 30 \sqrt{\varepsilon} r+60 \sqrt{\varepsilon} r \cdot E(r) \\
& \leq\left(900 \sqrt{\varepsilon} r^{2}+90 r+60 r E(r)\right) \sqrt{\varepsilon} \\
& \leq \delta \sqrt{\varepsilon}, \quad\left(\delta=10^{-5}\right) .
\end{aligned}
$$

Here we have used $r \leq r_{0}=10^{-10}$ and Lemma 5.5: $E(r) \leq 10^{-20}$. Thus we get

$$
\begin{aligned}
\left|f^{\prime}(z)\right|^{2} & \leq\left|a_{1}+3 a_{3} z^{2}\right|^{2}+E(r)^{2}+3 E(r)+\delta \sqrt{\varepsilon} \\
& \leq\left|a_{1}+3 a_{3} z^{2}\right|^{2}+4 E(r)+\delta \sqrt{\varepsilon} .
\end{aligned}
$$

Using Lemma 5.6, we have

$$
\begin{aligned}
\left|a_{1}+3 a_{3} z^{2}\right|^{2} & =\left|a_{1}\right|^{2}+2 \cdot 3 \cdot\left|a_{1}\right| \cdot\left|a_{3}\right| \cdot r^{2} \cos \left(2 \theta-\theta_{1}+\theta_{3}\right)+9\left|a_{3}\right|^{2} r^{4} \\
& \leq 1+2 \cdot 3\left|a_{3}\right| r^{2} \cos ^{+}\left(2 \theta-\theta_{1}+\theta_{3}\right)+9 \cdot 2^{6} r^{4} \\
& \leq 1+2\left(r^{2}+100 r^{3}+\delta \sqrt{\varepsilon}\right) \cos ^{+}\left(2 \theta-\theta_{1}+\theta_{3}\right)+576 r^{4} \\
& \leq 1+2 r^{2} \cos ^{+}\left(2 \theta-\theta_{1}+\theta_{3}\right)+200 r^{3}+2 \delta \cdot \sqrt{\varepsilon}+r^{3} \\
& =1+2 r^{2} \cos ^{+}\left(2 \theta-\theta_{1}+\theta_{3}\right)+201 r^{3}+2 \delta \cdot \sqrt{\varepsilon} .
\end{aligned}
$$

Thus

$$
\begin{aligned}
\left|f^{\prime}(z)\right|^{2} & \leq 1+2 r^{2} \cos ^{+}\left(2 \theta-\theta_{1}+\theta_{3}\right)+\left(201 r^{3}+4 E(r)\right)+3 \delta \cdot \sqrt{\varepsilon} \\
& \leq 1+2 r^{2} \cos ^{+}\left(2 \theta-\theta_{1}+\theta_{3}\right)+500 r^{3}+\frac{1}{2} \sqrt{\varepsilon} .
\end{aligned}
$$

Then we can estimate $|d f|^{2}(z)$ in the disk $r \leq r_{0}$. 
Proposition 5.8.

$|d f|^{2}(z) \leq 1-2 r^{2}\left(1-\cos ^{+}\left(2 \theta-\theta_{1}+\theta_{3}\right)\right)+600 r^{3}+\sqrt{\varepsilon} \quad\left(r \leq r_{0}\right)$.

Proof. To simplify the descriptions, we set $\varphi:=2 \theta-\theta_{1}+\theta_{3}$. From Lemma 5.7,

$$
\begin{aligned}
|d f|^{2}(z)=\frac{\left|f^{\prime}(z)\right|^{2}}{\left(1+|f(z)|^{2}\right)^{2}} & \leq \frac{1+2 r^{2} \cos ^{+} \varphi+500 r^{3}+\frac{1}{2} \sqrt{\varepsilon}}{\left(1+|f(z)|^{2}\right)^{2}} \\
& \leq \frac{1+2 r^{2} \cos ^{+} \varphi}{\left(1+|f(z)|^{2}\right)^{2}}+500 r^{3}+\frac{1}{2} \sqrt{\varepsilon}
\end{aligned}
$$

From Lemma 5.3,

$$
\begin{aligned}
\frac{1+2 r^{2} \cos ^{+} \varphi}{\left(1+|f(z)|^{2}\right)^{2} \leq} & \left(1+2 r^{2} \cos ^{+} \varphi\right)\left(1-2 r^{2}+30 r^{3}+\varepsilon\right) \\
= & 1-2 r^{2}\left(1-\cos ^{+} \varphi\right)+\left(30 r^{3}-4 r^{4} \cos ^{+} \varphi+60 r^{5} \cos ^{+} \varphi\right) \\
& +\varepsilon+2 \varepsilon r^{2} \cos ^{+} \varphi \\
\leq & 1-2 r^{2}\left(1-\cos ^{+} \varphi\right)+31 r^{3}+2 \varepsilon .
\end{aligned}
$$

Thus

$$
\begin{aligned}
|d f|^{2}(z) & \leq 1-2 r^{2}\left(1-\cos ^{+} \varphi\right)+531 r^{3}+\left(2 \sqrt{\varepsilon}+\frac{1}{2}\right) \sqrt{\varepsilon} \\
& \leq 1-2 r^{2}\left(1-\cos ^{+} \varphi\right)+600 r^{3}+\sqrt{\varepsilon} .
\end{aligned}
$$

The following proposition is the conclusion of this subsection.

Proposition 5.9. Let $D$ be a circular sector of radius $r_{0}$ and angle $\pi / 2$ centered at the origin in the complex plane, i.e.,

$$
D=\left\{r e^{\sqrt{-1} \theta} \in \mathbb{C} \mid 0 \leq r \leq r_{0}, \alpha \leq \theta \leq \alpha+\pi / 2\right\} \quad \text { for some } \alpha \in \mathbb{R} .
$$

Then

$$
\frac{1}{\operatorname{vol}(D)} \int_{D}|d f|^{2} d x d y \leq 1-\frac{1}{4} r_{0}^{2}+\sqrt{\varepsilon}<1-10^{-30}
$$




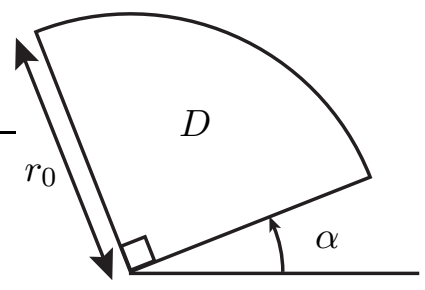

Figure 2: a circular sector $D$.

Proof. From Proposition 5.8,

$$
\begin{aligned}
& \frac{1}{\operatorname{vol}(D)} \int_{D}|d f|^{2} d x d y \\
& \leq 1+\sqrt{\varepsilon}-\frac{2}{\operatorname{vol}(D)} \int_{D} r^{2}\left(1-\cos ^{+}\left(2 \theta-\theta_{1}+\theta_{3}\right)\right)+\frac{600}{\operatorname{vol}(D)} \int_{D} r^{3}
\end{aligned}
$$

Since $\operatorname{vol}(D)=\pi r_{0}^{2} / 4$,

$$
\begin{aligned}
& \frac{1}{\operatorname{vol}(D)} \int_{D} r^{3}=\frac{4}{\pi r_{0}^{2}} \int_{0}^{r_{0}} r^{4} d r \int_{\alpha}^{\alpha+\pi / 2} d \theta=\frac{2}{5} r_{0}^{3} . \\
& \frac{1}{\operatorname{vol}(D)} \int_{D} r^{2}\left(1-\cos ^{+}\left(2 \theta-\theta_{1}+\theta_{3}\right)\right) \\
& =\frac{4}{\pi r_{0}^{2}} \int_{0}^{r_{0}} r^{3} d r \int_{\alpha}^{\alpha+\pi / 2}\left(1-\cos ^{+}\left(2 \theta-\theta_{1}+\theta_{3}\right)\right) d \theta \\
& =\frac{r_{0}^{2}}{\pi} \int_{\alpha}^{\alpha+\pi / 2}\left(1-\cos ^{+}\left(2 \theta-\theta_{1}+\theta_{3}\right)\right) d \theta .
\end{aligned}
$$

Setting $\varphi:=2 \theta-\theta_{1}+\theta_{3}$ and $\beta:=2 \alpha-\theta_{1}+\theta_{3}$, we have

$$
\begin{aligned}
\frac{r_{0}^{2}}{\pi} \int_{\alpha}^{\alpha+\pi / 2}\left(1-\cos ^{+}\left(2 \theta-\theta_{1}+\theta_{3}\right)\right) d \theta & =\frac{r_{0}^{2}}{2 \pi} \int_{\beta}^{\beta+\pi}\left(1-\cos ^{+} \varphi\right) d \varphi \\
& \geq \frac{r_{0}^{2}}{2 \pi} \int_{-\pi / 2}^{\pi / 2}\left(1-\cos ^{+} \varphi\right) d \varphi \\
& =r_{0}^{2}\left(\frac{1}{2}-\frac{1}{\pi}\right) .
\end{aligned}
$$


Thus

$$
\begin{aligned}
\frac{1}{\operatorname{vol}(D)} \int_{D}|d f|^{2} d x d y & \leq 1+\sqrt{\varepsilon}-\left(1-\frac{2}{\pi}\right) r_{0}^{2}+240 r_{0}^{3} \\
& \leq 1+\sqrt{\varepsilon}-\frac{1}{3} r_{0}^{2}+240 r_{0}^{3} \\
& \leq 1-\frac{1}{4} r_{0}^{2}+\sqrt{\varepsilon}
\end{aligned}
$$

Since $r_{0}=10^{-10}$ and $\varepsilon=10^{-100}$,

$$
1-\frac{1}{4} r_{0}^{2}+\sqrt{\varepsilon}=1-\frac{10^{-20}}{4}+10^{-50}<1-10^{-30} .
$$

\subsection{Proof of Theorem $\mathbf{1 . 4}$}

Let $f(z)$ be a meromorphic function in the complex plane which satisfies

$$
|d f|(z) \leq 1 \quad \text { for all } z \in \mathbb{C} .
$$

Here we don't suppose the condition (12) is satisfied. The following argument is similar to the argument in Subsection 2.2. First we establish a result on small squares.

Proposition 5.10. Let $K$ be a square of length of side $2 r_{0}$ in the complex plane. Then we have

$$
\frac{1}{\operatorname{vol}(K)} \int_{K}|d f|^{2} d x d y \leq 1-\varepsilon
$$

Proof. If $|d f|(z)<1-\varepsilon$ for all $z \in K$, it is obvious that

$$
\frac{1}{\operatorname{vol}(K)} \int_{K}|d f|^{2} d x d y \leq(1-\varepsilon)^{2}<1-\varepsilon .
$$

Hence we can suppose that there is a point $z_{0} \in K$ such that $|d f|\left(z_{0}\right) \geq 1-\varepsilon$. Since the length of side of $K$ is $2 r_{0}$, there is a circular sector $D$ of radius $r_{0}$ and angle $\pi / 2$ centered at $z_{0}$ such that $D \subset K$ (see Figure 3 ). We can suppose $z_{0}=0$ and $f\left(z_{0}\right)=0$ without loss of generality. Then $f(z)$ satisfies the condition (12). Applying Proposition 5.9 to this situation, we get

$$
\frac{1}{\operatorname{vol}(D)} \int_{D}|d f|^{2} d x d y \leq 1-10^{-30}
$$




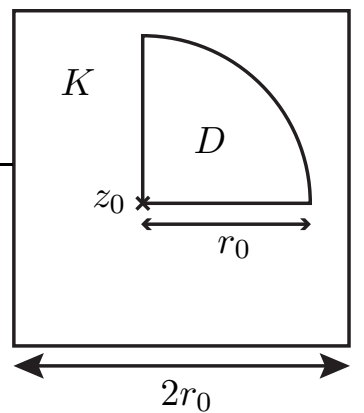

Figure 3: a circular sector $D$ in a square $K$.

$\operatorname{From} \operatorname{vol}(K)=4 r_{0}^{2}$ and $\operatorname{vol}(D)=\pi r_{0}^{2} / 4$,

$$
\frac{\operatorname{vol}(D)}{\operatorname{vol}(K)}=\frac{\pi}{16} .
$$

Using (14) and $|d f| \leq 1$, we get

$$
\begin{aligned}
& \frac{1}{\operatorname{vol}(K)} \int_{K}|d f|^{2} d x d y \\
& =\frac{\operatorname{vol}(D)}{\operatorname{vol}(K)} \cdot \frac{1}{\operatorname{vol}(D)} \int_{D}|d f|^{2} d x d y+\frac{1}{\operatorname{vol}(K)} \int_{K \backslash D}|d f|^{2} d x d y \\
& \leq \frac{\operatorname{vol}(D)}{\operatorname{vol}(K)}\left(1-10^{-30}\right)+\frac{1}{\operatorname{vol}(K)}(\operatorname{vol}(K)-\operatorname{vol}(D)) \\
& =1-\frac{\pi}{16} \cdot 10^{-30} \\
& <1-10^{-100}=1-\varepsilon .
\end{aligned}
$$

Proof of Theorem 1.4. We prove the theorem by packing squares of length of side $2 r_{0}$ in the disk $\bar{\Delta}(R)=\{z \in \mathbb{C}|| z \mid \leq R\}$. If we consider a tiling of the complex plane by squares of length of side $2 r_{0}$, then the disk $\bar{\Delta}\left(R-2 \sqrt{2} r_{0}\right)$ is covered by the squares contained in $\bar{\Delta}(R)$. And we can use the estimate of Proposition 5.10 on each squares of the tiling. Then the rest of the arguments are the same as the proof of Theorem 2.5. We omit the details. 


\section{§6. Proof of Theorem 1.7}

We define the $n+1$ hyperplanes $P_{0}, P_{1}, \ldots, P_{n}$ in $\mathbb{C} P^{n}$ by

$$
P_{i}: z_{i}=0 \quad(0 \leq i \leq n)
$$

where $\left[z_{0}: z_{1}: \cdots: z_{n}\right]$ is the homogeneous coordinate of $\mathbb{C} P^{n}$. Then

$$
\begin{aligned}
& \mathbb{C} P^{n} \backslash\left(P_{0} \cup \cdots \cup P_{n}\right) \\
& \quad=\left\{\left[1: z_{1}: z_{2}: \cdots: z_{n}\right] \mid z_{i} \neq 0(1 \leq i \leq n)\right\} \cong(\mathbb{C} \backslash\{0\})^{n} .
\end{aligned}
$$

The following proposition is proved in Berteloot-Duval [1, Appendice], and it is the key result for the proof of Theorem 1.7.

Proposition 6.1. Let $f: \mathbb{C} \rightarrow \mathbb{C} P^{n} \backslash\left(P_{0} \cup \cdots \cup P_{n}\right)$ be a holomorphic map with $\|d f\|_{\infty}<\infty$. Then there are complex numbers $a_{i}$ and $b_{i}(1 \leq i \leq n)$ such that

$$
f(z)=\left[1: \exp \left(a_{1} z+b_{1}\right): \exp \left(a_{2} z+b_{2}\right): \cdots: \exp \left(a_{n} z+b_{n}\right)\right] .
$$

I think this is a basic fact. So we will give a new proof of this proposition below. (In Tsukamoto [16] we prove a more general theorem.) We need:

Lemma 6.2. (i) Let $a$ and $b$ be complex numbers and set $f(z):=e^{a z+b}$. Then we have a positive constant $C$ such that

$$
\int_{|z| \leq r}|d f|^{2} d x d y \leq C r \quad \text { for all } r \geq 0 .
$$

Here $|d f|$ is the spherical derivative defined by

$$
|d f|(z)=\frac{1}{\sqrt{\pi}} \frac{\left|f^{\prime}(z)\right|}{1+|f(z)|^{2}} .
$$

(ii) Let $a, b, c$ be complex numbers with $a \neq 0$, and set $f(z):=e^{a z^{2}+b z+c}$. Then for any positive number $\varepsilon$, there exists an open subset $E$ in $[0,2 \pi]$ with $|E|<\varepsilon(|E|$ means the Lebesgue measure of $E)$ such that

$$
\int_{[0,2 \pi] \backslash E} d \theta \int_{0}^{\infty}|d f|^{2}\left(r e^{\sqrt{-1} \theta}\right) r d r<\infty .
$$


Proof. The proof of (i) is a direct calculation. We give the proof of (ii). Using the rotation of the coordinate, we can suppose that $a$ is a positive real number. From $f^{\prime}(z)=(2 a z+b) f(z)$, we have

$$
|d f|(z)=\frac{1}{\sqrt{\pi}} \frac{|2 a z+b||f(z)|}{1+|f(z)|^{2}}, \quad|f(z)|=\exp \left(\operatorname{Re}\left(a z^{2}+b z+c\right)\right) .
$$

We define $E \subset[0,2 \pi]$ by

$$
\begin{aligned}
E:= & \left(\frac{\pi}{4}-\frac{\delta}{2}, \frac{\pi}{4}+\frac{\delta}{2}\right) \cup\left(\frac{3 \pi}{4}-\frac{\delta}{2}, \frac{3 \pi}{4}+\frac{\delta}{2}\right) \\
& \cup\left(\frac{5 \pi}{4}-\frac{\delta}{2}, \frac{5 \pi}{4}+\frac{\delta}{2}\right) \cup\left(\frac{7 \pi}{4}-\frac{\delta}{2}, \frac{7 \pi}{4}+\frac{\delta}{2}\right) .
\end{aligned}
$$

Here $\delta$ is a sufficiently small positive number such that $|E|=4 \delta<\varepsilon$. Then

$$
|\cos 2 \theta| \geq \sin \delta \quad \text { for all } \theta \in[0,2 \pi] \backslash E .
$$

If $\cos 2 \theta \geq \sin \delta$, then we have

$$
\begin{aligned}
\operatorname{Re}\left(a z^{2}+b z+c\right) & =a r^{2} \cos 2 \theta+\operatorname{Re}(b z+c) \\
& \geq a(\sin \delta) r^{2}-|b| r-|c| .
\end{aligned}
$$

Hence

$$
\begin{aligned}
|d f|(z) & \leq \frac{1}{\sqrt{\pi}} \frac{|2 a z+b||f(z)|}{|f(z)|^{2}} \\
& \leq \frac{1}{\sqrt{\pi}}(2 a r+|b|) \exp \left(-\operatorname{Re}\left(a z^{2}+b z+c\right)\right) \\
& \leq \frac{1}{\sqrt{\pi}}(2 a r+|b|) \exp \left(-a(\sin \delta) r^{2}+|b| r+|c|\right) .
\end{aligned}
$$

If $\cos 2 \theta \leq-\sin \delta$, then a similar argument gives

$$
|d f|(z) \leq \frac{1}{\sqrt{\pi}}(2 a r+|b|) \exp \left(-a(\sin \delta) r^{2}+|b| r+|c|\right) .
$$

Therefore we get

$|d f|\left(r e^{\sqrt{-1} \theta}\right) \leq \frac{1}{\sqrt{\pi}}(2 a r+|b|) \exp \left(-a(\sin \delta) r^{2}+|b| r+|c|\right) \quad$ if $\theta \in[0,2 \pi] \backslash E$.

Thus we have a constant $C$ independent of $\theta$ such that

$$
\int_{0}^{\infty}|d f|^{2}\left(r e^{\sqrt{-1} \theta}\right) r d r \leq C \quad \text { for all } \theta \in[0,2 \pi] \backslash E .
$$


It follows that

$$
\int_{[0,2 \pi] \backslash E} d \theta \int_{0}^{\infty}|d f|^{2}\left(r e^{\sqrt{-1} \theta}\right) r d r \leq 2 \pi C<\infty .
$$

Proof of Proposition 6.1. ${ }^{3}$ From (15), we have holomorphic maps $f_{i}$ : $\mathbb{C} \rightarrow \mathbb{C} \backslash\{0\} \quad(1 \leq i \leq n)$ such that $f(z)=\left[1: f_{1}(z): \cdots: f_{n}(z)\right]$. There are holomorphic functions $g_{i}(z)$ in the complex plane such that $f_{i}(z)=$ $\exp \left(g_{i}(z)\right)(1 \leq i \leq n)$. We will prove that all $g_{i}(z)$ are linear functions. The proof falls into three steps. First we prove that $g_{i}(z)$ are polynomials. Next we show $\operatorname{deg}\left(g_{i}(z)\right) \leq 2$. In the last step we prove $\operatorname{deg}\left(g_{i}(z)\right) \leq 1$. The arguments in the first and second steps are standard in the Nevanlinna theory. The last step is a little tricky.

Set $m:=\|d f\|_{\infty}$. Here $|d f|^{2}=\frac{1}{4 \pi} \Delta \log \left(1+\sum\left|f_{i}\right|^{2}\right)$. Using Jensen's formula, we get

$$
\begin{aligned}
\frac{1}{4 \pi} \int_{|z|=r} \log \left(1+\sum_{i}\left|f_{i}\right|^{2}\right) d \theta & =\int_{1}^{r} \frac{d t}{t} \int_{|z| \leq t}|d f|^{2}(z) d x d y+\text { const } \\
& \leq \frac{1}{2} m^{2} \pi r^{2}+\text { const. }
\end{aligned}
$$

Set $\log ^{+} x=\max (0, \log x)$ for a non-negative real number $x$. We have

$$
\begin{aligned}
m\left(r, f_{i}\right):=\frac{1}{2 \pi} \int_{|z|=r} \log ^{+}\left|f_{i}\right| d \theta & \leq \frac{1}{4 \pi} \int_{|z|=r} \log \left(1+\sum_{j}\left|f_{j}\right|^{2}\right) d \theta \\
& \leq \frac{1}{2} m^{2} \pi r^{2}+\text { const. }
\end{aligned}
$$

From Nevanlinna's lemma on the logarithmic derivative,

$$
m\left(r, g_{i}^{\prime}\right)=m\left(r, f_{i}^{\prime} / f_{i}\right) \leq C\left(\log ^{+} m\left(r, f_{i}\right)+\log r\right) \leq \text { const } \cdot \log r+\text { const },
$$

for all $r \in[1, \infty) \backslash E$, where $E$ is a Lebesgue measurable set in $[1, \infty)$ with a finite measure. It follows that

$$
\liminf _{r \rightarrow \infty} \frac{m\left(r, g_{i}^{\prime}\right)}{\log r}<\infty .
$$

\footnotetext{
${ }^{3}$ This argument can be generalized to the proof of a more general theorem in Tsukamoto [16].
} 
This shows that $g_{i}^{\prime}(z)$ is a polynomial (this is a standard fact in the Nevanlinna theory). Hence $g_{i}(z)$ is also a polynomial. Next we will prove $\operatorname{deg}\left(g_{i}(z)\right) \leq 2$. Suppose $\operatorname{deg}\left(g_{1}(z)\right) \geq 3$. Then it is easy to see that there is a positive constant $C_{1}$ such that $m\left(r, f_{1}\right) \geq C_{1} r^{3}$ for $r \gg 0$. This means that

$$
C_{1} r^{3} \leq m\left(r, f_{1}\right) \leq \frac{1}{2} m^{2} \pi r^{2}+\text { const } \quad(r \gg 0)
$$

This is obviously impossible. Hence $\operatorname{deg}\left(g_{i}(z)\right) \leq 2(1 \leq i \leq n)$.

Finally we will prove $\operatorname{deg}\left(g_{i}(z)\right) \leq 1$. Suppose $\operatorname{deg}\left(g_{1}(z)\right)=2$. We have positive constants $C_{2}$ and $r_{0} \geq 1$ such that $m\left(r, f_{1}\right) \geq C_{2} r^{2}\left(r \geq r_{0}\right)$. From (16) and (17),

$$
C_{2} r^{2} \leq m\left(r, f_{1}\right) \leq \int_{1}^{r} \frac{d t}{t} \int_{|z| \leq t}|d f|^{2} d x d y+\text { const } \quad\left(r \geq r_{0}\right)
$$

From the definition of the Fubini-Study metric (1),

$$
\begin{aligned}
|d f|^{2} & =\frac{1}{\pi}\left[\frac{\sum_{i}\left|f_{i}^{\prime}\right|^{2}}{\left(1+\sum_{i}\left|f_{i}\right|^{2}\right)^{2}}+\frac{\sum_{i<j}\left|g_{i}^{\prime}-g_{j}^{\prime}\right|^{2}\left|f_{i}\right|^{2}\left|f_{j}\right|^{2}}{\left(1+\sum_{i}\left|f_{i}\right|^{2}\right)^{2}}\right] \\
& \leq \frac{1}{\pi}\left[\sum_{i} \frac{\left|f_{i}^{\prime}\right|^{2}}{\left(1+\left|f_{i}\right|^{2}\right)^{2}}+\sum_{i<j} \frac{\left|g_{i}^{\prime}-g_{j}^{\prime}\right|^{2}\left|f_{i}\right|^{2}\left|f_{j}\right|^{2}}{\left(\left|f_{i}\right|^{2}+\left|f_{j}\right|^{2}\right)^{2}}\right] \\
& =\frac{1}{\pi}\left[\sum_{i}\left|d f_{i}\right|^{2}+\sum_{i<j}\left|d\left(f_{i} / f_{j}\right)\right|^{2}\right] .
\end{aligned}
$$

$f_{i}(z)=\exp \left(g_{i}(z)\right), f_{i}(z) / f_{j}(z)=\exp \left(g_{i}(z)-g_{j}(z)\right)$, and the degrees of $g_{i}(z)$ and $g_{i}(z)-g_{j}(z)$ are at most two. Then we can apply Lemma 6.2 to holomorphic functions $f_{i}(z)$ and $f_{i}(z) / f_{j}(z)$; there are positive constants $C_{3}, C_{4}$ and a open set $F \subset[0,2 \pi]$ with $|F|<2 C_{2} / m^{2}$ such that

$$
\int_{[0,2 \pi] \backslash F} d \theta \int_{0}^{t}|d f|^{2}\left(r e^{\sqrt{-1} \theta}\right) r d r \leq C_{3} t+C_{4} .
$$


Then we have

$$
\begin{aligned}
& \int_{|z| \leq t}|d f|^{2} d x d y \\
& \quad=\int_{F} d \theta \int_{0}^{t}|d f|^{2}\left(r e^{\sqrt{-1} \theta}\right) r d r+\int_{[0,2 \pi] \backslash F} d \theta \int_{0}^{t}|d f|^{2}\left(r e^{\sqrt{-1} \theta}\right) r d r \\
& \quad \leq \int_{F} d \theta \int_{0}^{t} m^{2} r d r+C_{3} t+C_{4} \\
& =\frac{m^{2}|F|}{2} t^{2}+C_{3} t+C_{4} \\
& \leq C_{2} t^{2}+C_{3} t+C_{4} .
\end{aligned}
$$

Substituting this into (18), we get

$$
C_{2} r^{2} \leq \frac{C_{2}}{2} r^{2}+C_{3} r+C_{4} \log r+\text { const } \quad\left(r \geq r_{0}\right) .
$$

This is impossible. Thus we conclude that all $g_{i}(z)$ are linear functions.

Corollary 6.3. Let $f: \mathbb{C} \rightarrow \mathbb{C} P^{n} \backslash\left(P_{0} \cup \cdots \cup P_{n}\right)$ be a holomorphic map with $\|d f\|_{\infty}<\infty$. Then

$$
\limsup _{R \rightarrow \infty} \frac{1}{\pi R^{2}} \int_{|z| \leq R}|d f|^{2} d x d y=0 .
$$

Proof. From (19),

$$
|d f|^{2} \leq \frac{1}{\pi}\left[\sum_{i}\left|d f_{i}\right|^{2}+\sum_{i<j}\left|d\left(f_{i} / f_{j}\right)\right|^{2}\right] .
$$

Since $f_{i}(z)=\exp \left(g_{i}(z)\right)$ and $f_{i}(z) / f_{j}(z)=\exp \left(g_{i}(z)-g_{j}(z)\right)$ with linear functions $g_{i}(z)$ and $g_{i}(z)-g_{j}(z)$, we can apply Lemma 6.2 (i) and get

$$
\int_{|z| \leq R}|d f|^{2} d x d y \leq C R
$$

Here $C$ is a positive constant. Thus we get the conclusion.

Proof of Theorem 1.7. Using the defining equations of $H_{i}$ in (5), we define a biholomorphic map $A: \mathbb{C} P^{n} \rightarrow \mathbb{C} P^{n}$ by

$$
A\left(\left[z_{0}: z_{1}: \cdots: z_{n}\right]\right):=\left[\sum_{j} a_{0 j} z_{j}: \sum_{j} a_{1 j} z_{j}: \cdots: \sum_{j} a_{n j} z_{j}\right] .
$$


$A$ gives a biholomorphic map from $\mathbb{C} P^{n} \backslash\left(H_{0} \cup \cdots \cup H_{n}\right)$ to $\mathbb{C} P^{n} \backslash\left(P_{0} \cup\right.$ $\left.\cdots \cup P_{n}\right)$. We have a positive constant $C$ such that

$$
|d A(u)| \leq C|u| \text { and }\left|d A^{-1}(u)\right| \leq C|u| \text { for all } u \in T \mathbb{C} P^{n} .
$$

Let $f: \mathbb{C} \rightarrow \mathbb{C} P^{n} \backslash\left(H_{0} \cup \cdots \cup H_{n}\right)$ be a holomorphic map with $\|d f\|_{\infty} \leq$ 1. Then $A f$ is a holomorphic map from $\mathbb{C}$ to $\mathbb{C} P^{n} \backslash\left(P_{0} \cup \cdots \cup P_{n}\right)$ with $\|d(A f)\|_{\infty} \leq C<\infty$. Since $f=A^{-1} \circ A f$, Corollary 6.3 gives

$$
\begin{aligned}
\rho(f) & =\limsup _{R \rightarrow \infty} \frac{1}{\pi R^{2}} \int_{|z| \leq R}|d f|^{2} d x d y \\
& \leq \limsup _{R \rightarrow \infty} \frac{C^{2}}{\pi R^{2}} \int_{|z| \leq R}|d(A f)|^{2} d x d y=0 .
\end{aligned}
$$

Thus we conclude that

$$
\rho\left(\mathbb{C} P^{n} \backslash\left(H_{0} \cup \cdots \cup H_{n}\right)\right)=0 .
$$

\section{REFERENCES}

[1] F. Berteloot and J. Duval, Sur l'hyperbolicité de certains complémentaires, Enseign. Math., 47 (2001), 253-267.

[2] R. Brody, Compact manifolds and hyperbolicity, Trans. Amer. Math. Soc., 235 (1978), 213-219.

[3] E. Calabi, Isometric imbedding of complex manifolds, Ann. of Math., 58 (1953), 1-23.

[4] J. Clunie and W. K. Hayman, The spherical derivative of integral and meromorphic functions, Comment. Math. Helv., 40 (1966), 117-148.

[5] S. K. Donaldson and P. B. Kronheimer, The geometry of four-manifolds, Oxford University Press, New York, 1990.

[6] A. Eremenko, Normal holomorphic curves from parabolic regions to projective spaces, preprint, Purdue University (1998) (arXiv:0710.1281).

[7] M. Gromov, Topological invariants of dynamical systems and spaces of holomorphic maps: I, Math. Phys. Anal. Geom., 2 (1999), 323-415.

[8] S. Ji, Inequality for the distortion function of invertible sheaves on abelian varieties, Duke Math. J., 58 (1989), 657-667.

[9] G. R. Kempf, Metrics on invertible sheaves on abelian varieties, Topics in algebraic geometry (Guanajuato, 1989), Aportaciones Mat. Notas Investigación, 5, Soc. Mat. Mexicana, México, 1992, pp. 107-108.

[10] E. Lindenstrauss, Mean dimension, small entropy factors and an embedding theorem, Inst. Hautes Études Sci. Publ. Math., 89 (1999), 227-262. 
[11] E. Lindenstrauss and B. Weiss, Mean topological dimension, Israel J. Math., 115 (2000), 1-24.

[12] D. Minda, Yosida functions, Lectures on complex analysis (Chi-Tai Chuang, ed.), World Sci. Publishing, Singapore, 1988, pp. 197-213.

[13] D. Mumford, Tata lectures on Theta, I, Birkhäuser, Boston-Basel-Stuttgart, 1983.

[14] R. Nevanlinna, Zur Theorie der meromorphen Funktionen, Acta Math., 46 (1925), $1-99$.

[15] G. Tian, On a set of polarized Kähler metrics on algebraic manifolds, J. Differential Geom., 32 (1990), 99-130.

[16] M. Tsukamoto, On holomorphic curves in algebraic torus, J. Math. Kyoto Univ., 47-4 (2007), 881-892.

[17] M. Tsukamoto, Moduli space of Brody curves, energy and mean dimension, Nagoya Math. J., 192 (2008), 27-58.

[18] S. Zelditch, Szegö kernels and a theorem of Tian, Int. Math. Res. Notices, 6 (1998), $317-331$.

Department of Mathematics

Faculty of Science

Kyoto University

Kyoto 606-8502

Japan

tukamoto@math.kyoto-u.ac.jp 